\title{
A New Supply Chain System and Its Impulsive Synchronization
}

\author{
Yang Peng $D^{1},{ }^{1}$ Jiang Wu, ${ }^{1}$ Shiping Wen, ${ }^{2}$ Yuming Feng, ${ }^{3}$ Zhengwen Tu, ${ }^{3}$ and Limin Zou ${ }^{3}$ \\ ${ }^{1}$ School of Statistics, Southwestern University of Finance and Economics, Chengdu 611130, China \\ ${ }^{2}$ Centre for Artificial Intelligence, University of Technology Sydney, Ultimo, NSW 2007, Australia \\ ${ }^{3}$ Key Laboratory of Intelligent Information Processing and Control, Chongqing Three Gorges University, \\ Chongqing 404100, China \\ Correspondence should be addressed to Yang Peng; peng_yang2011@163.com
}

Received 23 July 2020; Revised 10 September 2020; Accepted 17 September 2020; Published 13 October 2020

Academic Editor: Quanxin Zhu

Copyright (c) 2020 Yang Peng et al. This is an open access article distributed under the Creative Commons Attribution License, which permits unrestricted use, distribution, and reproduction in any medium, provided the original work is properly cited.

\begin{abstract}
The purpose of this paper is to discuss modelling and synchronization of nonlinear supply chain system. Firstly, we present a new supply chain system which is sensitive to various uncertainties along with exogenous disturbances. Synchronization is an important method to reduce the negative impact of uncertainties and disturbances on the supply chain. Since impulsive control can reduce control cost and the amount of transmitted information drastically, we discuss impulsive synchronization behavior of two supply chain systems with the same structure. Finally, simulation experiments are given to show the effectiveness of our analytical results.
\end{abstract}

\section{Introduction}

Supply chain globalization is conducive to increasing the competitiveness of enterprises, but it also makes the supply chain face various uncertainties more frequently, such as demand uncertainty, supply uncertainty, and operational uncertainty. These uncertainties lead to the supply chain system showing complex dynamic characteristics, such as bullwhip effect $[1,2]$.

In recent years, academia and industry are actively studying the complexity of the supply chain system. For example, Anne et al. [3] presented a three-echelon supply chain model which can be stated as follows:

$$
\left\{\begin{array}{l}
\dot{x}_{1}=m x_{2}-(n+1) x_{1}, \\
\dot{x}_{2}=x_{1}\left(r-x_{3}\right)-x_{2}, \\
\dot{x}_{3}=x_{1} x_{2}+(b-1) x_{3},
\end{array}\right.
$$

where $x_{1}$ is the quantity demanded by retailer in current period, $x_{2}$ is the quantity distributors can supply in current period, and $x_{3}$ is the quantity produced in the current period depend on the order, $m$ is the coefficient of distributor delivery, $n$ is rate of customer demand satisfaction at retailer, $r$ is production threshold, and $b$ is manufacturer's safety stock factor. Supply chain system (1) has complex dynamic behavior. For example, if we choose $m=10, n=3, r=18$, and $b=3 / 7$, then system (1) is chaotic and Figure 1 shows the chaotic phenomenon of system (1) with the initial condition $\left(x_{1}, x_{2}, x_{3}\right)^{T}=(10,10,10)^{T}$.

Chaotic supply chain system (1) is very sensitive to disturbances. When a node in the supply chain changes, then the change will spread to the other two nodes quickly and cause the supply chain to deviate from the original state. For example, suppose that $m=10, n=3, r=18$, and $b=3 / 7$, if the state variables $X\left(t_{0}\right)=(10,10,10)$ becomes $Y\left(t_{0}\right)=(10.1,10,10)$ at some point, that is to say, the retailer's demand will increase by 0.1 unit, then the operation state of the supply chain will be changed, as shown in Figure 2.

From Figure 2, it can be seen that the small change of retailer's demand has little impact on the operation state of the supply chain at the beginning, but with the passage of time, the operation state of the supply chain will change greatly, resulting in the operation of the supply chain deviating from the preset state. Therefore, the general idea is to restore the deviated operation state to the expected track through appropriate control strategies, so as to reduce the impact of uncertainty factors. 


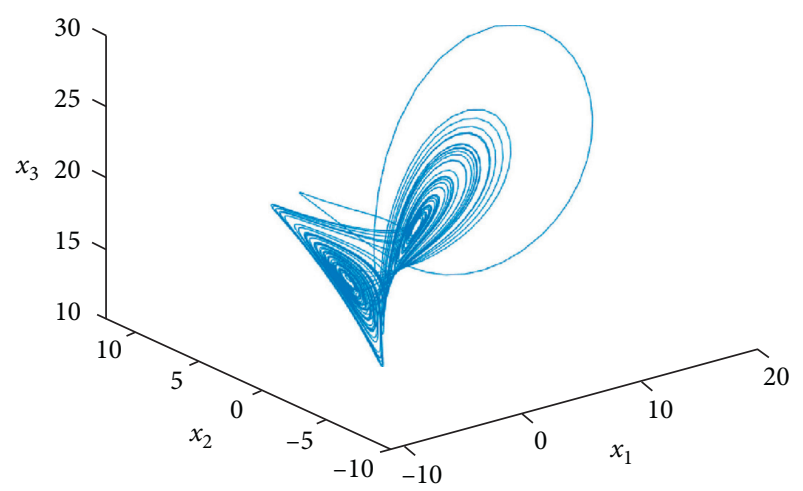

(a)

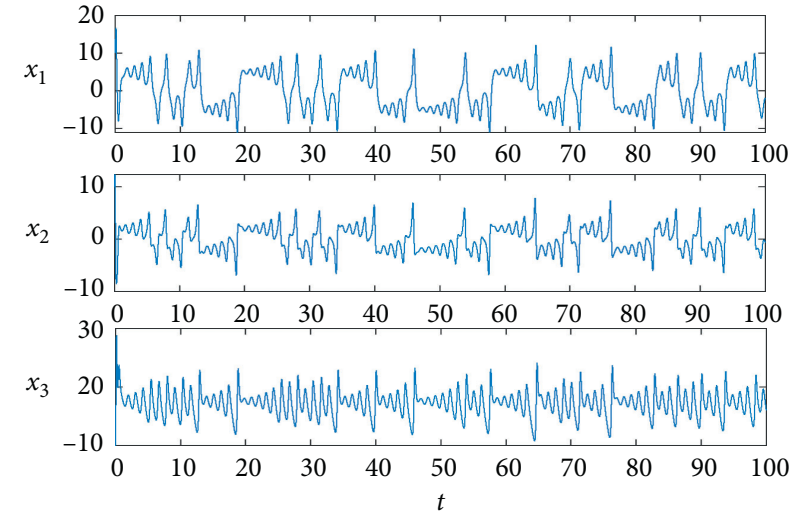

(b)

Figure 1: The chaotic phenomenon of system (1) with the initial condition $\left(x_{1}, x_{2}, x_{3}\right)^{T}=(10,10,10)^{T}$. (a) 3D phase portraits. (b) Time series of $x_{i}, i=1,2,3$.
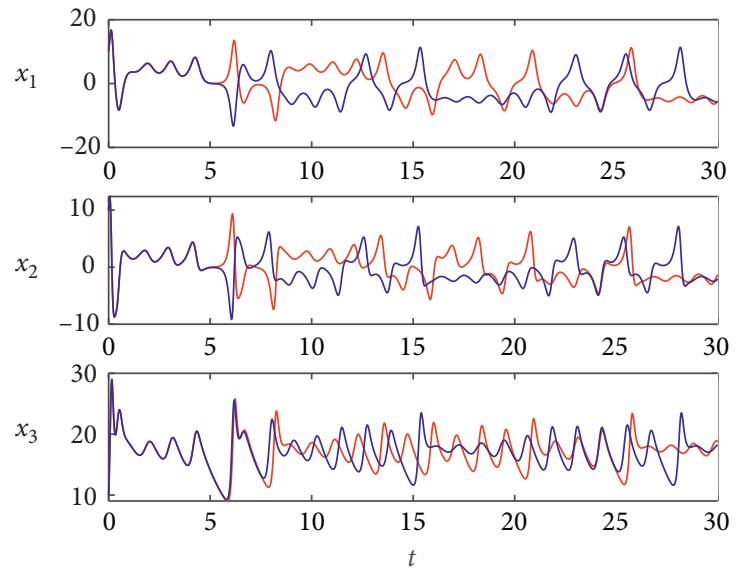

$-X$

$-Y$

(a)
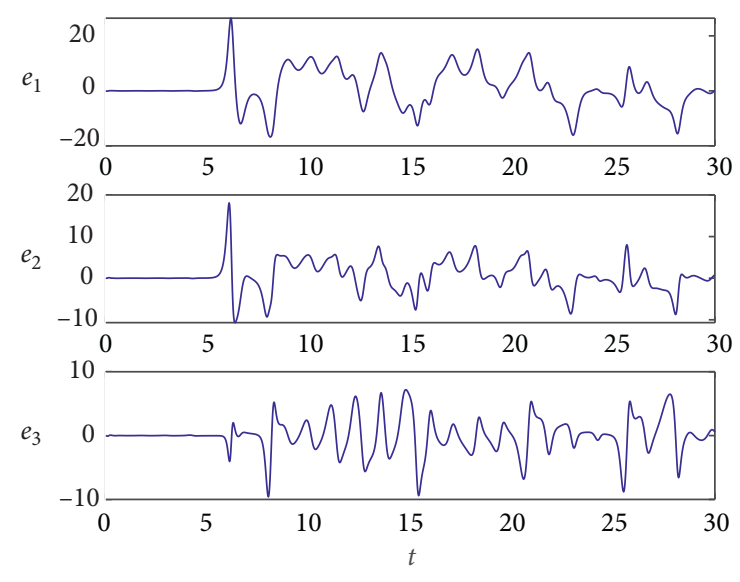

$-X$

$-Y$

Figure 2: The influence of uncertain factors on system (1). (a) Time series with different initial values. (b) Errors of each node.

Synchronization is an important method to reduce the negative impacts of uncertainties and disturbances on the supply chain. Göksu et al. [4] discussed the synchronization of two identical chaotic supply chain systems, and active and linear feedback controllers are applied for the system. Kocamaz et al. [5] presented the control of chaotic supply chain with Artificial Neural Network-based controllers and the synchronization of two identical chaotic supply chains. Tirandaz $[6,7]$ discussed adaptive modified projective synchronization and adaptive integral sliding mode control synchronization of supply chain system (1), respectively. Geng and Xiao [8] discussed the outer synchronization and parameter identification approach to the resilient recovery of supply network with uncertainty. Xu et al. [9] designed the adaptive supertwisting sliding mode control algorithm for chaos suppression and synchronization of the supply chain system.

Considering the fact that the demand of a product does not increase monotonically with the increase of inventory, Mondal [10] proposed a new supply chain model as follows:

$$
\left\{\begin{array}{l}
\dot{x}_{1}=\frac{m x_{2}}{1+a x_{2}}-(n+1) x_{1}, \\
\dot{x}_{2}=x_{1}\left(r-x_{3}\right)-\frac{p x_{2}}{1+a x_{2}}, \\
\dot{x}_{3}=x_{1} x_{2}+(b-1) x_{3},
\end{array}\right.
$$

where $a$ determines the saturation rate of demand with the increase of inventory and $p$ denotes the effect of demand variation of the inventory. The author also discussed synchronization behavior of two coupled identical supply chain models under both unidirectional and bidirectional coupling.

However, system (2) has a singularity when $x_{2}=-(1 / a)$ and the second equation is unreasonable according to the analysis of Anne et al. [3]. Therefore, in this paper, following the idea of Mondal, in which demand has a saturation level and it does not increase monotonically with the inventory, we will propose a new supply chain system which does not have 
singularity. In addition, considering the feasibility of the control strategy, we use the impulsive control method to realize the synchronization of the new system. For more information on impulsive control method and its advantages, the reader can refer to [11-22] and the references therein.

\section{A New Supply Chain System and Its Impulsive Synchronization}

Combining system (1) with (2), we propose a new supply chain system as follows:

$$
\left\{\begin{array}{l}
\dot{x}_{1}=\frac{m x_{2}}{\sqrt{1+a^{2} x_{2}^{2}}}-(n+1) x_{1}, \\
\dot{x}_{2}=x_{1}\left(r-x_{3}\right)-x_{2}, \\
\dot{x}_{3}=x_{1} x_{2}+(b-1) x_{3} .
\end{array}\right.
$$

Putting $a=0$ in system (3), we obtain system (1). The function $f\left(x_{2}\right)=\left(x_{2} /\left(\sqrt{1+a^{2} x_{2}^{2}}\right)\right)$ is used to describe the saturation degree of retailer's demand with respect to supply. It is easy to see that $f$ is odd function and $\lim _{x_{2} \longrightarrow+\infty} f\left(x_{2}\right)=(1 / a)$. The graph of function $f$ is shown in Figure 3.

By decomposing the linear and nonlinear parts of system in (3), we can rewrite it as follows:

$$
\dot{X}=\mathrm{AX}+\phi(X)
$$

where

$$
\begin{aligned}
A & =\left(\begin{array}{ccc}
-n-1 & 0 & 0 \\
r & -1 & 0 \\
0 & 0 & b-1
\end{array}\right), \\
X & =\left(\begin{array}{c}
x_{1} \\
x_{2} \\
x_{3}
\end{array}\right), \\
\phi(X) & =\left(\begin{array}{c}
\frac{m x_{2}}{\sqrt{1+a^{2} x_{2}^{2}}} \\
-x_{1} x_{3} \\
x_{1} x_{2}
\end{array}\right) .
\end{aligned}
$$

If we choose $m=10, n=3, r=18, b=3 / 7$, and $a=0.3$, then system (3) is chaotic and Figure 4 shows the chaotic phenomenon of system (3) with the initial condition $\left(x_{1}, x_{2}, x_{3}\right)^{T}=(10,10,10)^{T}$.

Next, we investigate impulsive synchronization of two supply chain systems with the same structure. System (3) is the driving system and the driven system is defined as

$$
\left\{\begin{array}{l}
\frac{\mathrm{d} Y}{\mathrm{~d} t}=\mathrm{AY}+\phi(Y), \quad t \neq \tau_{k}, \\
\Delta Y=\mathrm{Be}, \quad t=\tau_{k}, k=1,2, \ldots,
\end{array}\right.
$$

where $Y=\left(y_{1}, y_{2}, y_{3}\right)^{T}$ and $B$ is impulsive control gain matrix. The symbol $e$ denotes system errors and

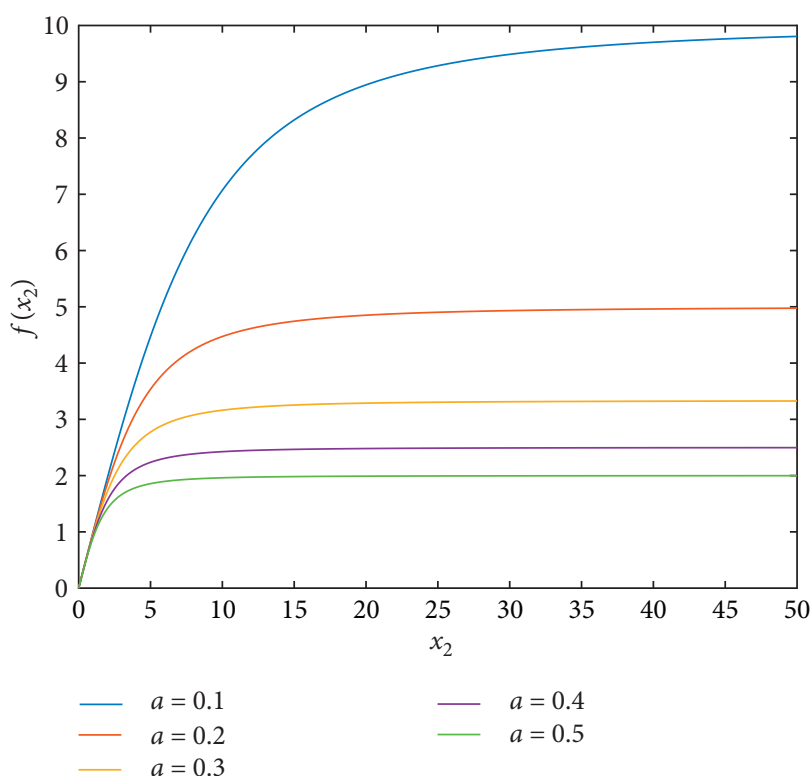

FIgURE 3: The graph of function $f$ for different values of $a$.

$e=\left(e_{1}, e_{2}, e_{3}\right)^{T}=\left(y_{1}-x_{1}, y_{2}-x_{2}, y_{3}-x_{3}\right)^{T}$. There will be a jump in system (6) when $t=\tau_{k}$ and $t_{0}=\tau_{0}<\tau_{1}<\tau_{2}<, \ldots, \lim _{k \rightarrow \infty} \tau_{k}=\infty$. Through, system (6) minus system (3), we obtain

$$
\begin{cases}\frac{\mathrm{d} e}{\mathrm{~d} t}=\mathrm{Ae}+\psi(X, Y), & t \neq \tau_{k}, \\ \Delta e=\mathrm{Be}, & t=\tau_{k}, k=1,2, \ldots,\end{cases}
$$

where $\psi(X, Y)=\phi(Y)-\phi(X)$.

Due to the boundedness of the chaotic system (3), we suppose that $\left|x_{i}\right| \leq M, i=1,2,3$. In the following theorem, we present a sufficient condition for the stability of error system (7).

Theorem 1. Let $\alpha$ and $\lambda$ be the largest eigenvalues of $A^{T}+A$ and $\left(I+B^{T}\right)(I+B)$, respectively. If

$$
\log \lambda+(\alpha+2 M+3 m)\left(\tau_{k}-\tau_{k-1}\right)<0
$$

holds for all $k=1,2, \ldots$, then the origin of impulsive synchronization error system (7) is asymptotically stable.

Proof. Let

$$
V(e(t))=e^{T}(t) e(t) .
$$

For $t \in\left[t_{0}, \tau_{1}\right)$, we have

$$
\begin{aligned}
\frac{\mathrm{d} V(e(t))}{\mathrm{d} t} & =(A e+\psi(X, Y))^{T} e+e^{T}(A e+\psi(X, Y)) \\
& =e^{T} A^{T} e+\psi(X, Y)^{T} e+e^{T} A e+e^{T} \psi(X, Y) \\
& =e^{T}\left(A^{T}+A\right) e+2 e^{T} \psi(X, Y) .
\end{aligned}
$$




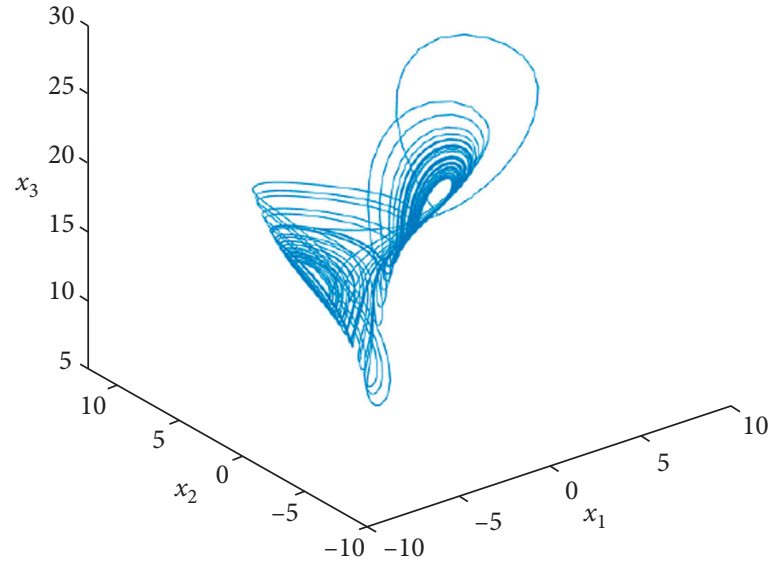

(a)
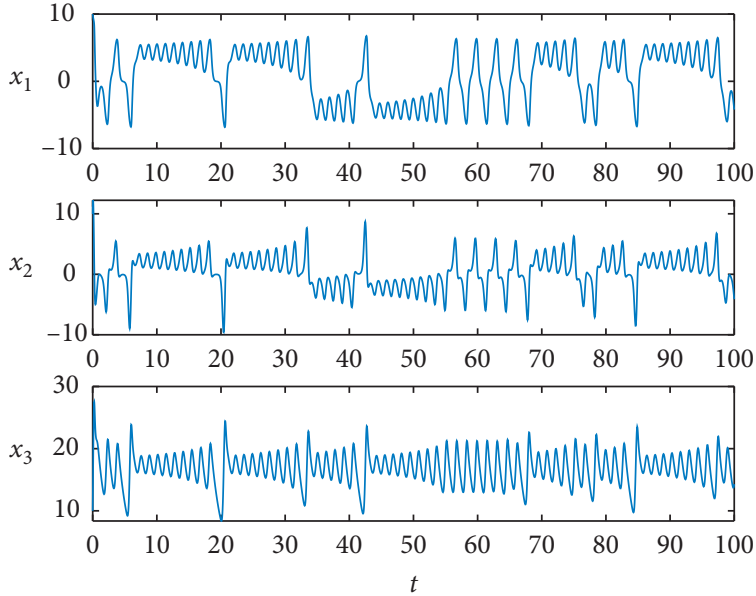

(b)

FIgUre 4: The chaotic phenomenon of system (3) with the initial condition $\left(x_{1}, x_{2}, x_{3}\right)^{T}=(10,10,10)^{T}$. (a) 3D phase portraits. (b) Time series of $x_{i}, i=1,2,3$.

By the arithmetic-geometric mean inequality, we have

$$
\begin{aligned}
& e^{T} \psi(X, Y)=\left(y_{1}-x_{1}, y_{2}-x_{2}, y_{3}-x_{3}\right)\left(\frac{m y_{2}}{\sqrt{1+a^{2} y_{2}^{2}}}-\frac{m x_{2}}{\sqrt{1+a^{2} x_{2}^{2}}},-y_{1} y_{3}+x_{1} x_{3}, y_{1} y_{2}-x_{1} x_{2}\right)^{T} \\
& =e_{1}\left(\frac{m y_{2}}{\sqrt{1+a^{2} y_{2}^{2}}}-\frac{m x_{2}}{\sqrt{1+a^{2} y_{2}^{2}}}+\frac{m x_{2}}{\sqrt{1+a^{2} y_{2}^{2}}}-\frac{m x_{2}}{\sqrt{1+a^{2} x_{2}^{2}}}\right)+x_{1} x_{3} y_{2}+x_{2} y_{1} y_{3}-y_{3} x_{1} x_{2}-x_{3} y_{1} y_{2} \\
& =e_{1}\left(\frac{m e_{2}}{\sqrt{1+a^{2} y_{2}^{2}}}+m x_{2}\left(\frac{1}{\sqrt{1+a^{2} y_{2}^{2}}}-\frac{1}{\sqrt{1+a^{2} x_{2}^{2}}}\right)\right)+x_{2} e_{1} e_{3}-x_{3} e_{1} e_{2} \\
& =e_{1}\left(\frac{m e_{2}}{\sqrt{1+a^{2} y_{2}^{2}}}+m x_{2}\left(\frac{a^{2}\left(x_{2}-y_{2}\right)\left(x_{2}+y_{2}\right)}{\sqrt{1+a^{2} y_{2}^{2}} \sqrt{1+a^{2} x_{2}^{2}}\left(\sqrt{1+a^{2} x_{2}^{2}}+\sqrt{1+a^{2} y_{2}^{2}}\right)}\right)\right)+x_{2} e_{1} e_{3}-x_{3} e_{1} e_{2} \\
& =\frac{m e_{1} e_{2}}{\sqrt{1+a^{2} y_{2}^{2}}}\left(1-a^{2}\left(\frac{x_{2}}{\sqrt{1+a^{2} x_{2}^{2}}} \frac{x_{2}+y_{2}}{\sqrt{1+a^{2} x_{2}^{2}}+\sqrt{1+a^{2} y_{2}^{2}}}\right)\right)+x_{2} e_{1} e_{3}-x_{3} e_{1} e_{2} \\
& \leq \frac{m e_{1} e_{2}}{\sqrt{1+a^{2} y_{2}^{2}}}\left(1+a^{2}\left(\left|\frac{x_{2}}{\sqrt{1+a^{2} x_{2}^{2}}}\right|\left|\frac{x_{2}+y_{2}}{\sqrt{1+a^{2} x_{2}^{2}}+\sqrt{1+a^{2} y_{2}^{2}}}\right|\right)\right)+\left|x_{2} e_{1} e_{3}\right|+\left|x_{3} e_{1} e_{2}\right| \\
& \leq \frac{m e_{1} e_{2}}{\sqrt{1+a^{2} y_{2}^{2}}}\left(1+a^{2}\left(\left|\frac{1}{a}\right|\left|\frac{2}{a}\right|\right)\right)+M\left(\left|e_{1} e_{3}\right|+\left|e_{1} e_{2}\right|\right) \\
& \leq \frac{3 m e_{1} e_{2}}{\sqrt{1+a^{2} y_{2}^{2}}}+M\left(\left|e_{1} e_{3}\right|+\left|e_{1} e_{2}\right|\right) \\
& \leq \frac{3}{2} m\left(e_{1}^{2}+e_{2}^{2}\right)+M\left(\left|e_{1} e_{3}\right|+\left|e_{1} e_{2}\right|\right) \\
& \leq \frac{3 m+2 M}{2} e^{T} e .
\end{aligned}
$$


It follows from (10) and (11) that

$$
\frac{\mathrm{d} V(e(t))}{\mathrm{d} t} \leq(\alpha+2 M+3 m) V(e(t)) .
$$

Let

$$
g(t)=\frac{\mathrm{d} V(e(t))}{\mathrm{d} t}-(\alpha+2 M+3 m) V(e(t)) .
$$

Then, we obtain

$$
V(e(t))=e^{(\alpha+2 M+3 m)\left(t-t_{0}\right)}\left(\int_{t_{0}}^{t} e^{-(\alpha+2 M+3 m) \tau} g(\tau) d \tau+V\left(e\left(t_{0}\right)\right)\right) .
$$

Since $g(t) \leq 0$, we have

$$
V(e(t)) \leq V\left(e\left(t_{0}\right)\right) e^{(\alpha+2 M+3 m)\left(t-t_{0}\right)},
$$

for $t \in\left[t_{0}, \tau_{1}\right)$.

When $t=\tau_{1}$, we have

$$
\begin{aligned}
V\left(e\left(\tau_{1}\right)\right) & =\left((I+B) e\left(\tau_{1}^{-}\right)\right)^{T}(I+B) e\left(\tau_{1}^{-}\right) \\
& =e^{T}\left(\tau_{1}^{-}\right)\left(I+B^{T}\right)(I+B) e\left(\tau_{1}^{-}\right) \\
& \leq \lambda e^{T}\left(\tau_{1}^{-}\right) e\left(\tau_{1}^{-}\right) \\
& =\lambda V\left(e\left(\tau_{1}^{-}\right)\right) .
\end{aligned}
$$

If $t \in\left[\tau_{1}, \tau_{2}\right)$, then repeating the process of deriving inequality (15), we obtain

$$
V(e(t)) \leq V\left(e\left(\tau_{1}\right)\right) e^{(\alpha+2 M+3 m)\left(t-\tau_{1}\right)} .
$$

It follows from (16) and (17) that

$$
V(e(t)) \leq \lambda V\left(e\left(\tau_{1}^{-}\right)\right) e^{(\alpha+2 M+3 m)\left(t-\tau_{1}\right)} .
$$

By inequality (18) and local order preserving of function limit, we obtain

$$
V\left(e\left(\tau_{1}^{-}\right)\right) \leq V\left(e\left(t_{0}\right)\right) e^{(\alpha+2 M+3 m)\left(\tau_{1}-t_{0}\right)} .
$$

Combining with inequalities (18) and (19), we have

$$
V(e(t)) \leq \lambda V\left(e\left(t_{0}\right)\right) e^{(\alpha+2 M+3 m)\left(t-t_{0}\right)},
$$

for $t \in\left[\tau_{1}, \tau_{2}\right)$.

When $t=\tau_{2}$, by the same method which is used to deduce inequality (16), we obtain

$$
V\left(e\left(\tau_{2}\right)\right) \leq \lambda V\left(e\left(\tau_{2}^{-}\right)\right),
$$

and so

$$
\begin{aligned}
V(e(t)) & \leq V\left(e\left(\tau_{2}\right)\right) e^{(\alpha+2 M+3 m)\left(t-\tau_{2}\right)} \\
& \leq \lambda V\left(e\left(\tau_{2}^{-}\right)\right) e^{(\alpha+2 M+3 m)\left(t-\tau_{2}\right)} \\
& \leq \lambda\left(\lambda V\left(e\left(t_{0}\right)\right) e^{(\alpha+2 M+3 m)\left(\tau_{2}-t_{0}\right)}\right) e^{(\alpha+2 M+3 m)\left(t-\tau_{2}\right)} \\
& =\lambda^{2} V\left(e\left(t_{0}\right)\right) e^{(\alpha+2 M+3 m)\left(t-t_{0}\right)},
\end{aligned}
$$

for $t \in\left[\tau_{2}, \tau_{3}\right)$.

Therefore, by the repeatability of the proof process and inequalities (15), (20), and (22), we know that

$$
\begin{aligned}
V(e(t)) & \leq \lambda^{k-1} V\left(e\left(t_{0}\right)\right) e^{(\alpha+2 M+3 m)\left(t-t_{0}\right)} \\
& =V\left(e\left(t_{0}\right)\right) e^{(\alpha+2 M+3 m)\left(t-t_{0}\right)} e^{(k-1) \log \lambda} \\
& =V\left(e\left(t_{0}\right)\right) e^{\log \lambda+(\alpha+2 M+3 m)\left(\tau_{1}-t_{0}\right)} e^{\log \lambda+(\alpha+2 M+3 m)\left(\tau_{2}-\tau_{1}\right)} \cdots e^{(\alpha+2 M+3 m)\left(t-\tau_{k-1}\right)} \\
& =\lambda^{-1} V\left(e\left(t_{0}\right)\right) e^{\log \lambda+(\alpha+2 M+3 m)\left(\tau_{1}-t_{0}\right)} e^{\log \lambda+(\alpha+2 M+3 m)\left(\tau_{2}-\tau_{1}\right)} \cdots e^{\log \lambda+(\alpha+2 M+3 m)\left(t-\tau_{k-1}\right)},
\end{aligned}
$$

for $t \in\left[\tau_{k-1}, \tau_{k}\right)$.

So, if $\alpha+2 M+3 m<0$, then

$$
V(e(t)) \leq V\left(e\left(t_{0}\right)\right) e^{\sum_{i=1}^{k-1}\left(\log \lambda+(\alpha+2 M+3 m)\left(\tau_{i}-\tau_{i-1}\right)\right)} .
$$

On the other hand, if $\alpha+2 M+3 m \geq 0$, then

$$
V(e(t)) \leq \lambda^{-1} V\left(e\left(t_{0}\right)\right) e^{\sum_{i=1}^{k}\left(\log \lambda+(\alpha+2 M+3 m)\left(\tau_{i}-\tau_{i-1}\right)\right)} .
$$

Hence, if $\log \lambda+(\alpha+2 M+3 m)\left(\tau_{k}-\tau_{k-1}\right)<0$, $k=1,2, \ldots$, then $V(e(t)) \longrightarrow 0$ when $k \longrightarrow+\infty$, that is, $t \longrightarrow+\infty$. This completes the proof.

In generally, the intensity of each impulsive may not be the same, in view of this, we have the following result.
Theorem 2. Let $\alpha$ and $\lambda_{k}$ be the largest eigenvalues of $A^{T}+A$ and $\left(I+B_{k}^{T}\right)\left(I+B_{k}\right)$, respectively. If

$$
\log \lambda_{k}+(\alpha+2 M+3 m)\left(\tau_{k}-\tau_{k-1}\right)<0,
$$

holds for all $k=1,2, \ldots$, then the origin of impulsive synchronization error system (7) is asymptotically stable. The proof of this result is the same as Theorem 1.

Recently, $\mathrm{Hu}$ et al. considered the effect of noise disturbances and provided some new methods [23-26]. Now, following the ideas of these authors, we consider system (3) with parameter uncertainty and state variables perturbation. The corresponding system can be described as follows:

$$
\frac{\mathrm{d} X}{\mathrm{~d} t}=(A+\Delta A) X+\phi(X)+f(X)
$$


where $f(X)=\left(a \sin \left(x_{1}\right), b \cos \left(x_{2}\right), c \sin \left(x_{3}\right)\right)^{T}$ and

$$
\Delta A=\left(\begin{array}{ccc}
\delta n & 0 & 0 \\
\delta r & 0 & 0 \\
0 & 0 & \delta b
\end{array}\right)
$$

Similarly, we can obtain the error system as follows:

$$
\begin{cases}\frac{\mathrm{d} e}{\mathrm{~d} t}=(A+\Delta A) e+\psi(X, Y)+g(X, Y), & t \neq \tau_{k}, \\ \Delta e=B e, & t=\tau_{k}, k=1,2, \ldots,\end{cases}
$$

where $g(X, Y)=f(Y)-f(X)$.

Theorem 3. Let $\alpha$ and $\lambda$ be the largest eigenvalues of $A^{T}+A$ and $\left(I+B^{T}\right)(I+B)$, respectively. Let $\gamma=\max \left\{\delta_{1}, \delta_{2}\right\}$ with $\delta_{1}=2 \delta_{b}, \delta_{2}=\delta_{n}+\sqrt{\delta_{n}^{2}+\delta_{r}^{2}}$, and $\rho=2 \max \{|a|,|b|,|c|\}$. If

$$
\ln \lambda+(\alpha+\gamma+2 M+3 m+\rho)\left(\tau_{i}-\tau_{i-1}\right)<0
$$

holds for all $k=1,2, \ldots$, then the origin of impulsive synchronization error system (29) is asymptotically stable.
Proof. Note that

$$
\Delta A+(\Delta A)^{T}=\left(\begin{array}{ccc}
2 \delta_{n} & \delta_{r} & 0 \\
\delta_{r} & 0 & 0 \\
0 & 0 & 2 \delta_{b}
\end{array}\right)
$$

Then, we have

$$
\begin{aligned}
\left|x I-\Delta A+(\Delta A)^{T}\right| & =\left|\begin{array}{ccc}
x-2 \delta_{n} & \delta_{r} & 0 \\
\delta_{r} & x & 0 \\
0 & 0 & x-2 \delta_{b}
\end{array}\right| \\
& =\left(x-2 \delta_{b}\right)\left[x\left(x-2 \delta_{n}\right)-\delta_{r}^{2}\right] \\
& =\left(x-2 \delta_{b}\right)\left(x^{2}-2 \delta_{n} x-\delta_{r}^{2}\right) .
\end{aligned}
$$

So, the eigenvalues of $\Delta A+(\Delta A)^{T}$ are

$$
\begin{aligned}
\delta_{1} & =2 \delta_{b}, \\
\delta_{2,3} & =\delta_{n} \pm \sqrt{\delta_{n}^{2}+\delta_{r}^{2}} .
\end{aligned}
$$

Meanwhile, simple calculations show that

$$
\begin{aligned}
e^{T} g(X, Y) & =\left(a e_{1}\left(\sin \left(Y_{1}\right)-\sin \left(X_{1}\right)\right)+b e_{2}\left(\cos \left(Y_{2}\right)-\cos \left(X_{2}\right)\right)+c e_{3}\left(\sin \left(Y_{3}\right)-\sin \left(X_{3}\right)\right)\right) \\
& \leq\left(|a| e_{1}\left(Y_{1}-X_{1}\right)+|b| e_{2}\left(Y_{2}-X_{2}\right)+|c| e_{3}\left(Y_{3}-X_{3}\right)\right) \\
& =\left(|a| e_{1}^{2}+|b| e_{2}^{2}+|c| e_{3}^{2}\right) \\
& \leq \max \{|a|,|b|,|c|\} e^{T} e .
\end{aligned}
$$

Let us construct the following Lyapunov function:

For $t \in\left[t_{0}, \tau_{1}\right)$, we have

$$
V(e(t))=e^{T} e .
$$

$$
\begin{aligned}
\frac{\mathrm{d} V(e(t))}{\mathrm{d} t} & =\left((A+\Delta A) e+\psi(X, Y)+g(X, Y)^{T}\right) e+e^{T}((A+\Delta A) e+\psi(X, Y)+g(X, Y)) e \\
& =e^{T}\left(A+A^{T}+\Delta A+\Delta A^{T}\right) e+2 e^{T} \psi(X, Y)+2 e^{T} g(X, Y) .
\end{aligned}
$$

It follows from (11) and (34) that

$$
\begin{aligned}
\frac{\mathrm{d} V(e(t))}{\mathrm{d} t} & \leq e^{T}\left(A+A^{T}+\Delta A+\Delta A^{T}\right) e+(2 M+3 m) e^{T} e+\rho e^{T} e \\
& \leq(\alpha+\gamma) e^{T} e+(2 M+3 m) e^{T} e+\rho e^{T} e \\
& =(\alpha+\gamma+2 M+3 m+\rho) .
\end{aligned}
$$

The rest of proof is the same as Theorem 1. This completes the proof.

\section{Simulation Experiments}

In this section, we will use some numerical simulation experiments to illustrate how to use the strategies presented in Section 2 to eliminate or suppress the negative impacts of uncertain factors on the supply chain system.

Experiment 1. This example simulates the impact of the change of retailer demand on the system operation in supply chain system (3) and restores the operation state of supply chain to the expected track through impulsive control strategy. As mentioned above, if we choose $m=10, n=3, r=18, b=$ $3 / 7$, and $a=0.3$, then system (3) is chaotic. 

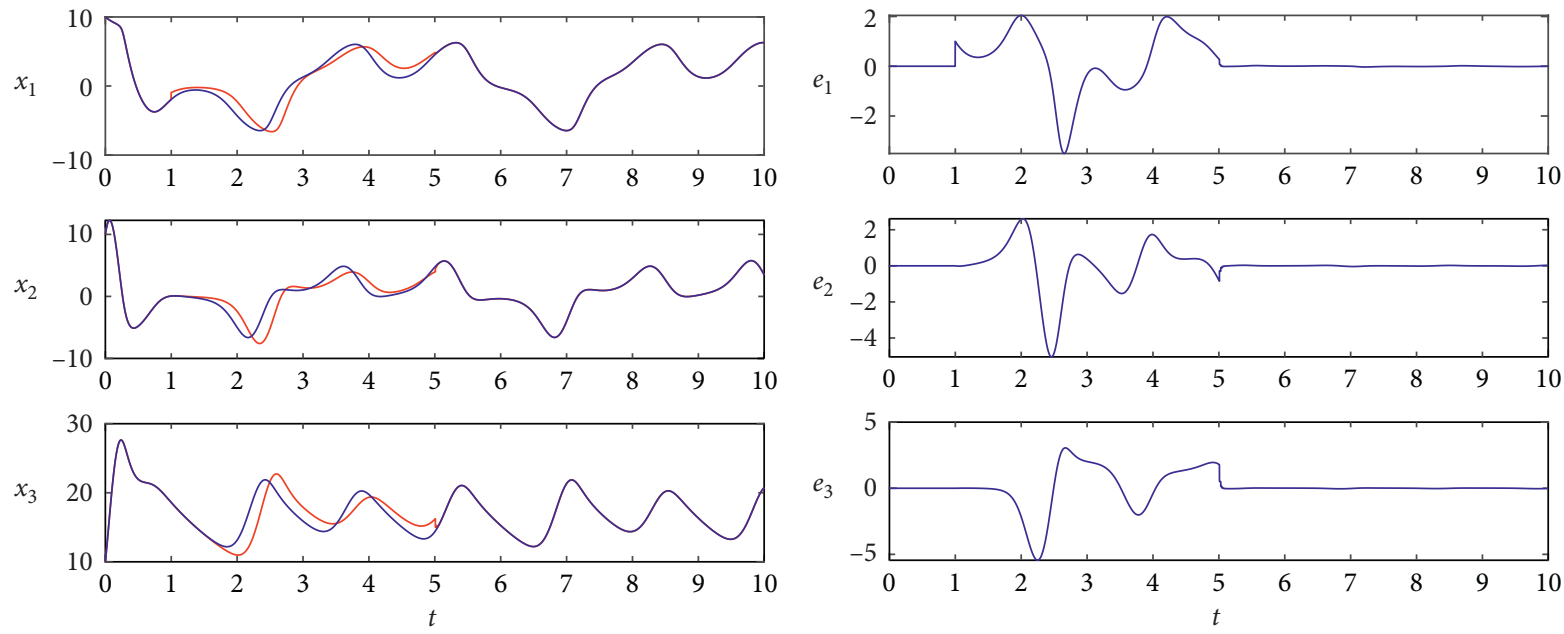

— Disturbed operation state

_ Expected operation state

(a)

(b)

FIGURE 5: Impulsive synchronization of supply chain system (3). (a) Time series of $x_{i}, i=1,2$, 3. (b) Errors of each node.

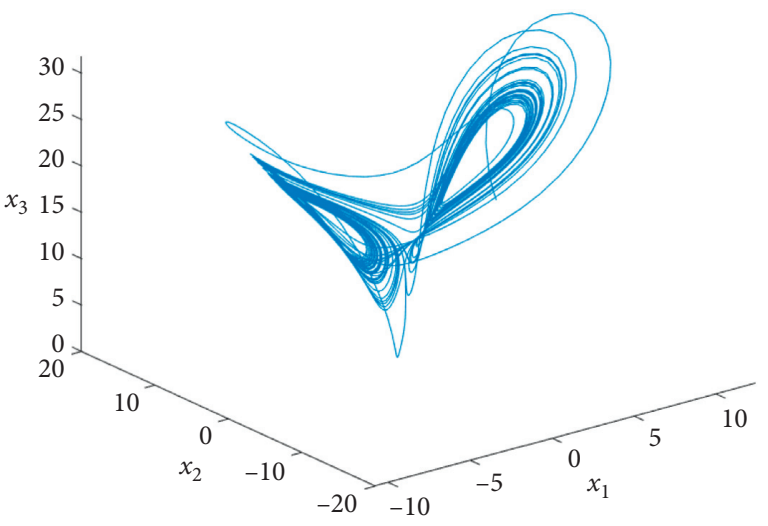

(a)
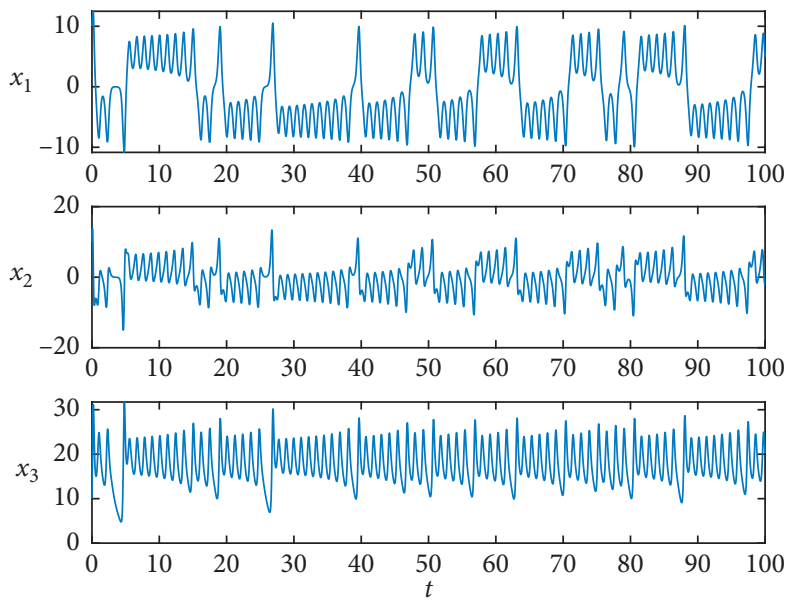

(b)

Figure 6: The chaotic phenomenon of system (27) with the initial condition $X\left(t_{0}\right)=(10,10,10)$. (a) $3 \mathrm{D}$ phase portraits. (b) Time series of $x_{i}$, $i=1,2,3$.

Small calculations show that $\alpha=13.2483$ and $M=$ 27.5293. When $t=1$, the retailer's demand is reduced by one unit. We find that the operation of the supply chain is seriously deviated from the expected state soon afterwards. We hope that the supply chain system can return to its original state by itself after a period. Unfortunately, the two tracks have not come together. We start to implement impulsive control when $t=5$ and assume that the intensity of each regulation is the same. According to Theorem 1, by simple calculations, we know that if the intensity and time interval of impulsive control satisfy the following inequality

$$
\log \lambda+98.3069\left(\tau_{k}-\tau_{k-1}\right)<0,
$$

then, the disturbed operation state will return to the expected orbit after appropriate impulsive control. In order to satisfy inequality (38), we choose impulsive control gain matrix $B=-0.7 I$, that is, the value of each node in the supply chain $Y_{i+1}$ becomes $0.7 X_{i}+0.3 Y_{i}$. So, the time interval of regulation should meet $\tau_{k}-\tau_{k-1}<0.0245$. Suppose that $\tau_{k}-\tau_{k-1}=0.0200$, which means that the time interval of impulsive regulation is 20 unit time (the unit time is taken as 0.001 in the experiment). The numerical simulation results are shown in Figure 5. 

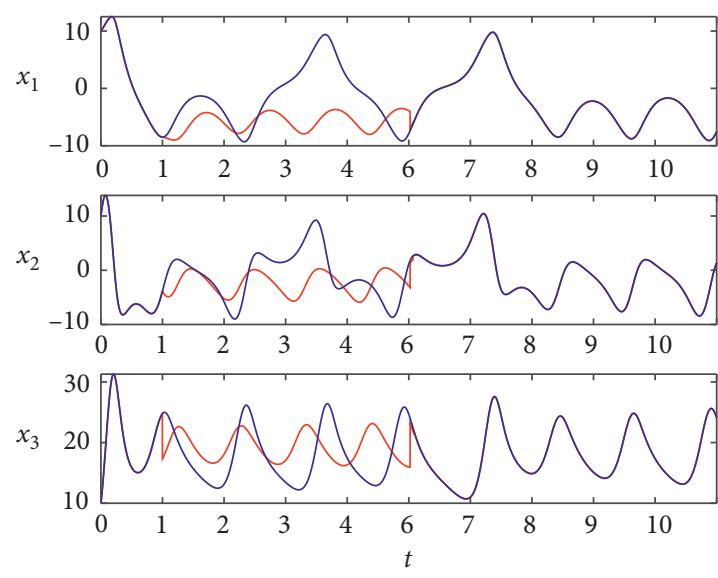

- Disturbed operation state

_ Expected operation state

(a)

FIGURE 7: Impulsive synchronization of supply chain system

Experiment 2. In this example, we simulate the impact of the change of the manufacturer's output on the system operation in the supply chain system (27), and through impulsive control strategy, the supply chain operation state is restored to the expected track. Let $m=5, n=1, r=20, b=0.3, a=$ $0.1, \delta_{m}=\delta_{n}=0.1, \delta_{r}=0.2$, and $\delta_{b}=-0.1$ and $f(t, X)=$ $\left(0.01 \sin \left(x_{1}\right), 0.02 \cos \left(x_{2}\right), 0.03 \sin \left(x_{3}\right)\right)^{T}$ be input disturbance. Suppose that $X\left(t_{0}\right)=(10,10,10)$, the phase diagram of the system and state trajectory of each node are shown in Figure 6.

Small calculations show that $\alpha=17.0250, M=$ $31.7329, \gamma=0.3236$, and $\rho=0.06$. When $t=1$, the produced quantity of the manufacturer is reduced, say, 0.7 times of the original for some reason. We find that the operation of the supply chain is seriously deviated from the expected state soon afterwards. We start to implement impulsive control when $t=15$ and assume that the intensity of each regulation is the same. According to Theorem 3, by simple calculations we know that if the intensity and time interval of impulsive control satisfy the following inequality

$$
\log \lambda+96.1744\left(\tau_{k}-\tau_{k-1}\right)<0,
$$

then, the disturbed operation state will return to the expected orbit after appropriate impulsive control. In order to satisfy inequality (39), we choose impulsive control gain matrix $B=-0.9 I$, that is, the value of each node in the supply chain $Y_{i+1}$ becomes $0.9 X_{i}+0.1 Y_{i}$. So, the time interval of regulation should meet $\tau_{k}-\tau_{k-1}<0.0479$ and suppose that $\tau_{k}-\tau_{k-1}=0.0450$ which means that the time interval of impulsive regulation is 45 unit time (the unit time is taken as 0.001 in the experiment). The numerical simulation results are shown in Figure 7.

It can be seen from Figure 7 that the operation state of the system quickly returns to the expected orbit.
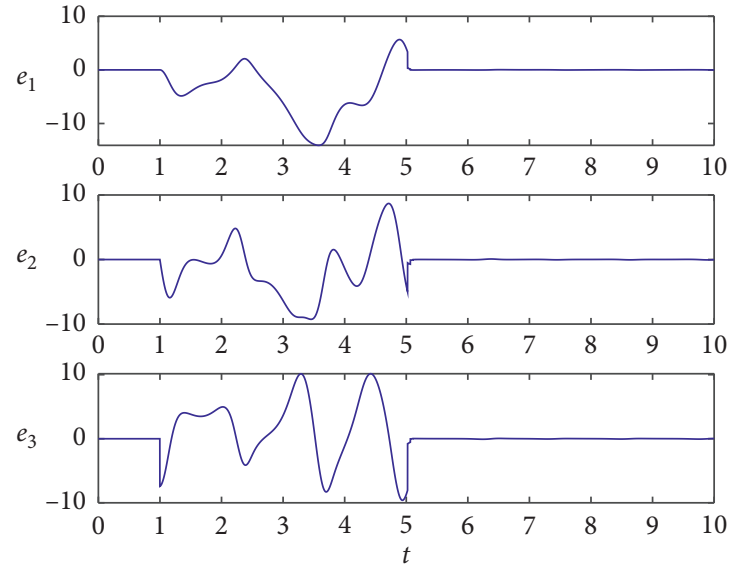

(b)

(27). (a) Time series of $x_{i}, i=1,2,3$. (b) Errors of each node.

\section{Conclusion}

As pointed out in [3], in today's global marketplace, the supply chain is dynamic and unstable. This dynamic and unstable properties produce a variety of uncertainties in the supply chain, such as demand uncertainty, supply uncertainty, delivery uncertainty, and forecast uncertainty. These uncertainties make the upstream and downstream transmission of the supply chain complex and nonlinear. In this paper, we propose a new supply chain system which is more in line with the actual situation. As stated earlier, synchronization is an important way to avoid uncertainty in supply chains, and impulsive control is an effective method to realize synchronization. So, we also discuss impulsive synchronization of supply chain system and some impulsive synchronization strategies are obtained. These results may be useful to the management of the supply chain.

\section{Data Availability}

The Matlab code data used to support the findings of this study are available from the corresponding author upon request.

\section{Conflicts of Interest}

The authors declare that they have no conflicts of interest.

\section{Authors' Contributions}

All authors contributed equally to the writing of this paper. All authors read and approved the final version of this paper.

\section{Acknowledgments}

This research was supported by the Fundamental Research Funds for the Central Universities (Grant no. JBK2002011) and Science and Technology Research Program of 
Chongqing Municipal Education Commission (Grant no. KJQN201800811).

\section{References}

[1] Q. Hu, "Bullwhip effect in a supply chain model with multiple delivery delays," Operations Research Letters, vol. 47, no. 1, pp. 36-40, 2019.

[2] E. Pastore, A. Alfieri, G. Zotteri, and J. E. Boylan, "The impact of demand parameter uncertainty on the bullwhip effect," European Journal of Operational Research, vol. 283, no. 1, pp. 94-107, 2020.

[3] K. R. Anne, J. C. Chedjou, and K. Kyamakya, "Bifurcation analysis and synchronisation issues in a three-echelon supply chain," International Journal of Logistics Research and Applications, vol. 12, no. 5, pp. 347-362, 2009.

[4] A. Göksu, U. E. Kocamaz, and Y. Uyaroğlu, "Synchronization and control of chaos in supply chain management," Computers \& Industrial Engineering, vol. 86, pp. 107-115, 2015.

[5] U. E. Kocamaz, H. Taşkın, Y. Uyaroğlu, and A. Göksu, "Control and synchronization of chaotic supply chains using intelligent approaches," Computers \& Industrial Engineering, vol. 102, pp. 476-487, 2016.

[6] H. Tirandaz, "On adaptive modified projective synchronization of a supply chain management system," PramanaJournal of Physics, vol. 89, no. 6, p. 85, 2017.

[7] H. Tirandaz, "Adaptive integral sliding mode control method for synchronization of supply chain system," WSEAS Transactions on Systems and Control, vol. 13, pp. 54-62, 2018.

[8] L. Geng and R. Xiao, "Outer synchronization and parameter identification approach to the resilient recovery of supply network with uncertainty," Physica A: Statistical Mechanics and Its Applications, vol. 482, pp. 407-421, 2017.

[9] X. Xu, S.-D. Lee, H.-S. Kim, and S.-S. You, "Management and optimisation of chaotic supply chain system using adaptive sliding mode control algorithm," International Journal of Production Research, pp. 1-7, 2020, In press.

[10] S. Mondal, "A new supply chain model and its synchronization behaviour," Chaos, Solitons \& Fractals, vol. 123, pp. 140-148, 2019.

[11] C. Li, X. Liao, and X. Zhang, "Impulsive synchronization of chaotic systems," Chaos: An Interdisciplinary Journal of Nonlinear Science, vol. 15, no. 2, Article ID 023104, 2005.

[12] X. Li and J. Wu, "Stability of nonlinear differential systems with state-dependent delayed impulses," Automatica, vol. 64, pp. 63-69, 2016.

[13] X. Li and J. Cao, "An impulsive delay inequality involving unbounded time-varying delay and applications," IEEE Transactions on Automatic Control, vol. 62, no. 7, pp. 36183625, 2017.

[14] X. Li and S. Song, "Stabilization of delay systems: delay-dependent impulsive control," IEEE Transactions on Automatic Control, vol. 62, no. 1, pp. 406-411, 2017.

[15] Q. Song, H. Yan, Z. Zhao, and Y. Liu, "Global exponential stability of complex-valued neural networks with both timevarying delays and impulsive effects," Neural Networks, vol. 79, pp. 108-116, 2016.

[16] Q. Song, H. Yan, Z. Zhao, and Y. Liu, "Global exponential stability of impulsive complex-valued neural networks with both asynchronous time-varying and continuously distributed delays," Neural Networks, vol. 81, pp. 1-10, 2016.

[17] W. Xiong, D. Zhang, and J. Cao, "Impulsive synchronisation of singular hybrid coupled networks with time-varying nonlinear perturbation," International Journal of Systems Science, vol. 48, no. 2, pp. 417-424, 2017.

[18] F. Yao, J. Cao, P. Cheng, and L. Qiu, "Generalized average dwell time approach to stability and input-to-state stability of hybrid impulsive stochastic differential systems," Nonlinear Analysis: Hybrid Systems, vol. 22, pp. 147-160, 2016.

[19] X. Yang and J. Cao, "Hybrid adaptive and impulsive synchronization of uncertain complex networks with delays and general uncertain perturbations," Applied Mathematics and Computation, vol. 227, pp. 480-493, 2014.

[20] X. Yang, J. Cao, and Z. Yang, "Synchronization of coupled reaction-diffusion neural networks with time-varying delays via pinning-impulsive controller," SIAM Journal on Control and Optimization, vol. 51, no. 5, pp. 3486-3510, 2013.

[21] X. Yang, J. Lam, D. W. C. Ho, and Z. Feng, "Fixed-time synchronization of complex networks with impulsive effects via nonchattering control," IEEE Transactions on Automatic Control, vol. 62, no. 11, pp. 5511-5521, 2017.

[22] X. Yang, D. Peng, X. Lv, and X. Li, "Recent progress in impulsive control systems," Mathematics and Computers in Simulation, vol. 155, pp. 244-268, 2019.

[23] W. Hu, H. R. Q. Zhu, and H. R. Karimi, "Some improved Razumikhin stability criteria for impulsive stochastic delay differential systems," IEEE Transactions on Automatic Control, vol. 64, no. 12, pp. 5207-5213, 2019.

[24] W. Hu and Q. Zhu, "Stability analysis of impulsive stochastic delayed differential systems with unbounded delays," Systems \& Control Letters, vol. 136, Article ID 104606, 2020.

[25] W. Hu, H. R. Q. Zhu, and H. R. Karimi, "On the pth moment integral input-to-state stability and input-to-state stability criteria for impulsive stochastic functional differential equations," International Journal of Robust and Nonlinear Control, vol. 29, no. 16, pp. 5609-5620, 2019.

[26] Q. Zhu, “pth moment exponential stability of impulsive stochastic functional differential equations with Markovian switching," Journal of the Franklin Institute, vol. 351, no. 7, pp. 3965-3986, 2014. 